\title{
DESIGN AND TESTING OF A 2K SUPERFLUID HELIUM HEAT STATION*
}

\author{
W. R. Hicks, E. F. Daly, J. Preble, M. Wiseman, C. Rode \\ Thomas Jefferson National Accelerator Facility \\ Newport News, VA 23606, US
}

\begin{abstract}
Three transitional cryomodules (SL21, FEL03, Renascence) have been constructed as part of an energy upgrade effort at Thomas Jefferson National Accelerator Facility (JLab). Each transitional cryomodule contains eight superconducting radio-frequency (SRF) cavities. Within the vacuum vessel, waveguides transmit up to $13 \mathrm{~kW}$ of RF power to the superconducting niobium cavities. The waveguides also provide the thermal transition between the room temperature ceramic RF window and the niobium fundamental power coupler (FPC), a 300K temperature gradient across $\sim 20 \mathrm{~cm}$ ! The thermal performance of the waveguides is determined in part by the placement of heat stations and bellows. The original $13 \mathrm{~kW}$ waveguide design incorporated a single $60 \mathrm{~K}$ heat station and two bellows resulting in a total heat load (static + dynamic) to the FPC of $\sim 3 \mathrm{~W}$ per waveguide. To minimize this heat load and stabilize the FPC temperatures, a $2 \mathrm{~K}$ superfluid helium heat station design was incorporated into the second transitional cryomodule, FEL03, installed in the JLab Free Electron Laser (FEL). The designed heat station is capable of removing up to $1.12 \mathrm{~W}$, with a bath temperature of $2.05 \mathrm{~K}$, while remaining sub-lambda. This paper describes the design, analysis and testing of the heat station.
\end{abstract}

\section{INTRODUCTION}

The Continuous Electron Beam Accelerator Facility (CEBAF) at JLab houses two superconducting linear accelerators (linacs). Each linac has slots for 25 cryomodules. Currently there are 20 cryomodules per linac. Each cryomodule contains eight SRF cavities. Copper plated stainless steel half-height waveguides are used to transmit RF power to the superconducting niobium cavities. The waveguides also provide the thermal transition between the ceramic windows and FPCs. The waveguide design is optimized thermally to minimize the heat load to the FPC. The main contributors to the thermal performance of the waveguides are copper plating, heat stations and bellows. Selecting an

*This work was supported by DOE contract DE-AC05-84ER40150 Modification No. M175 
appropriate plating thickness and locating heat stations and bellows appropriately will thermally optimize the waveguide design for a given RF power. The original CEBAF design incorporates a $60 \mathrm{~K}$ heat station and two bellows. The calculated static and dynamic heat loads for the SL21 (south linac zone 21) warm/cold waveguide transitions are .4W and $\sim 3 \mathrm{~W}$, respectively.

During commissioning of the first transitional cryomodule, SL21, in the CEBAF machine, pressure oscillations to .0418atm, liquid level instabilities of .5-2\%, and unstable FPC temperatures, $>9.1 \mathrm{~K}$, were observed. The pressure oscillations and liquid level instabilities were the result of an undersized helium vessel/return header transition. The waveguide heat load was one possible cause of the unstable FPC temperatures. As a result, $2 \mathrm{~K}$ heat stations were added to the waveguides in the FEL03 cryomodule already under construction.

\section{MECHANICAL DESIGN}

The $2 \mathrm{~K}$ heat station design was driven by existing space constraints. The design consists of two $1.3 \mathrm{~cm}(1 / 2 \mathrm{inch})$ stainless steel tubes soldered to the broad wall of the waveguide transition. The cooling tubes were flattened to form a racetrack-shaped cross section. The flat surfaces of the tubes provide soldering surfaces, and increased contact area for heat transfer. Flattening the tubes was also necessary to clear existing flange hardware. The cooling tubes were plumbed into the primary helium circuit. The bottom of the heat station is connected to the $1.9 \mathrm{~cm}(3 / 4$ inch IPS) helium supply header via $1.9 \mathrm{~cm}(3 / 4$ inch IPS) piping. The top of the heat station is connected to the $12.7 \mathrm{~cm}$ (5 inch IPS) helium return header by a series of $1.9 \mathrm{~cm}$ ( $3 / 4$ inch IPS), $2.5 \mathrm{~cm}$ ( 1 inch IPS), and $3.8 \mathrm{~cm}\left(1 \frac{1}{2}\right.$ inch IPS) piping. Braided flex-line and bellows were incorporated to accommodate alignment. Figure 1 shows a solid model of the primary helium circuit including the $2 \mathrm{~K}$ heat station and waveguide assembly.

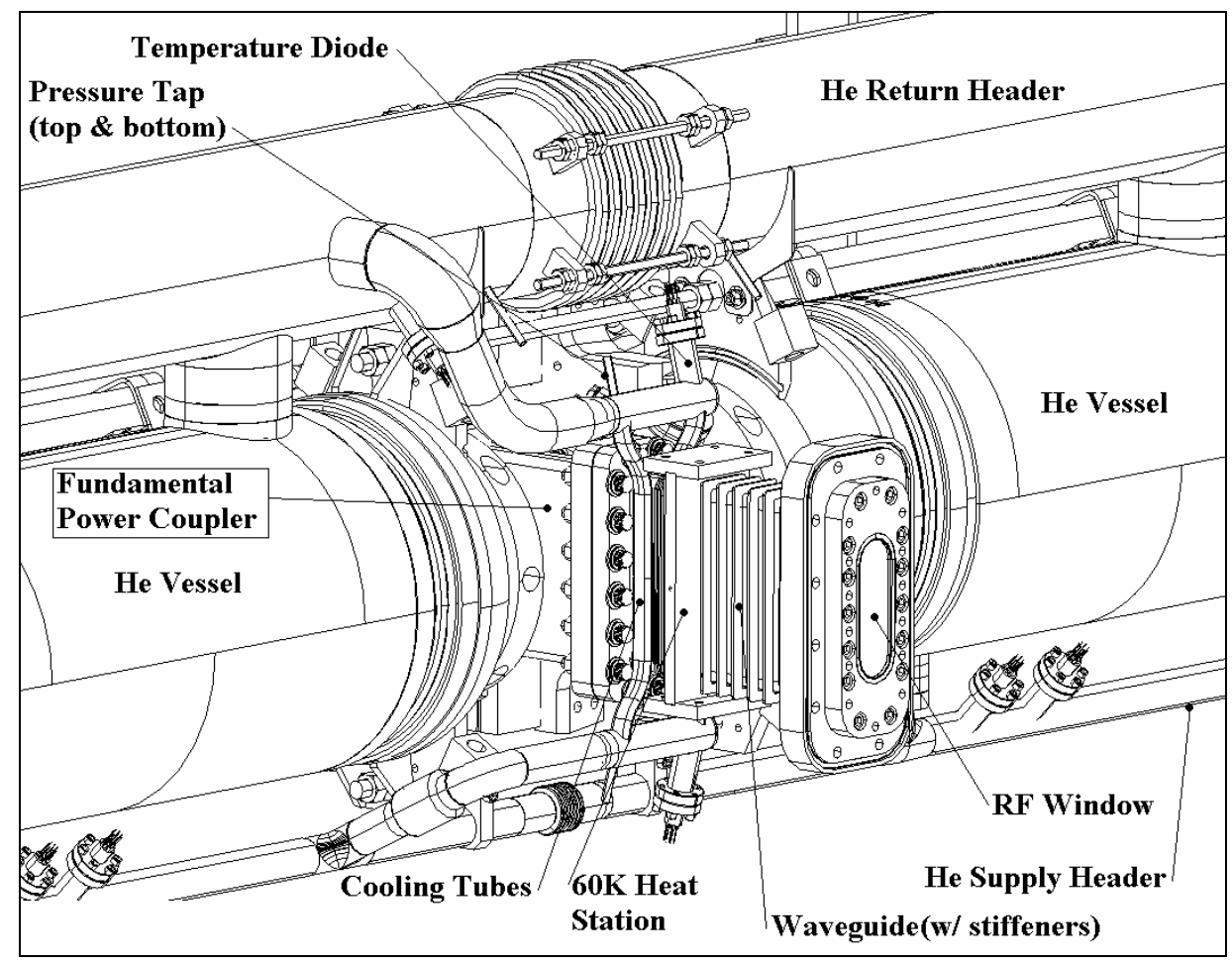

FIGURE 1. Solid model of the primary helium circuit including the waveguide assembly and heat station. 


\section{BACKGROUND}

Below the $\lambda$-transition, $\mathrm{T}_{\lambda}=2.172 \mathrm{~K}$, at saturated vapor pressure, helium exists as He II. The thermal conductivity of He II is extremely high, as large as $86,500 \mathrm{~W} / \mathrm{m}-\mathrm{K}$. The thermal conductivity of He I is $\sim .024 \mathrm{~W} / \mathrm{m}-\mathrm{K}$ [1]. The importance of staying below the $\lambda$ transition becomes obvious when comparing the thermal conductivities of He I and He II.

Of primary interest when using He II in this application is the critical heat flux. If the He II temperature rises above the $\lambda$-transition or the critical heat flux is exceeded, boiling can occur and He I and/or vapor may form [2]. Knowing the cryogenic system operating and transition temperatures, the critical heat flux can be found. The amount of heat that can be removed by a channel is limited by either a radial $\left(\mathrm{q}_{\mathrm{o}}{ }^{*}\right)$ or longitudinal $\left(\mathrm{q}^{*}\right)$ heat flux. As a point of reference, these two quantities can be found for this design by solving the following two equations [2].

$$
\begin{gathered}
q_{o}^{*}=\left(\frac{2 \cdot \psi}{r_{o}} \int_{T_{b}}^{T^{*}} \frac{d T}{f(T)}\right)^{\frac{1}{3}}=1.73 \frac{W}{\mathrm{~cm}_{(\text {SurfaceArea })}^{2}} \\
q^{*}=\left(\frac{1}{L} \int_{T_{b}}^{T_{\lambda}} \frac{d T}{f(T)}\right)^{\frac{1}{3}}=.85 \frac{W}{\mathrm{~cm}_{(\text {CrossSectionalArea })}^{2}}
\end{gathered}
$$

In equations (1) and (2):

$\mathrm{L}=$ length of cooling tube in thermal contact with waveguide $(11.63 \mathrm{~cm})$

$f^{1}(\mathrm{~T})=$ heat conductivity function $\left(\mathrm{W}^{3} / \mathrm{m}^{5}-\mathrm{K}\right)$

$\mathrm{T}_{\mathrm{b}}=$ cryogenic system operating temperature $(2.1 \mathrm{~K})$

$\mathrm{T}_{\lambda}=$ transition temperature $(2.172 \mathrm{~K})$

$\mathrm{T}^{\prime}=\mathrm{T}_{\mathrm{b}}+.01 \mathrm{~K}$

$\psi=$ radius dependent quantity $<1$

$\mathrm{r}_{\mathrm{o}}=$ inside radius of cooling channel $(.43 \mathrm{~cm})$.

\section{ANALYSIS}

The purpose of the analysis is to determine the critical heat flux for the given heat station geometry. RF surface currents create joule heating on the interior copper plated surfaces of the waveguide. Heat flows into the $2 \mathrm{~K}$ heat station along the length of the cooling tubes that are soldered to the waveguide where it is conducted away by He II. The critical heat flux is determined by the temperature difference between the He II in the cooling tube, at $\mathrm{T}_{\lambda}$, and the bath temperature, $\mathrm{T}_{\mathrm{b}}$, in the headers.

Equation (2) defines the critical heat flux for a heat input into one end of a channel at $T_{\lambda}$ with heat flow to the other end of the channel at $T_{b}$ [2]. Equation (2) accurately describes the critical heat flux for tube sections R3 and R4 in figure 2. The next step in the analysis was determining the critical heat flow into the straight portion of the cooling tube, R2 in figure 2. For this section there is a uniform heat flux along the length of the channel, therefore equation (2) is not appropriate. By applying the conservation of energy, defining a differential control volume, and identifying the appropriate heat fluxes, a differential 


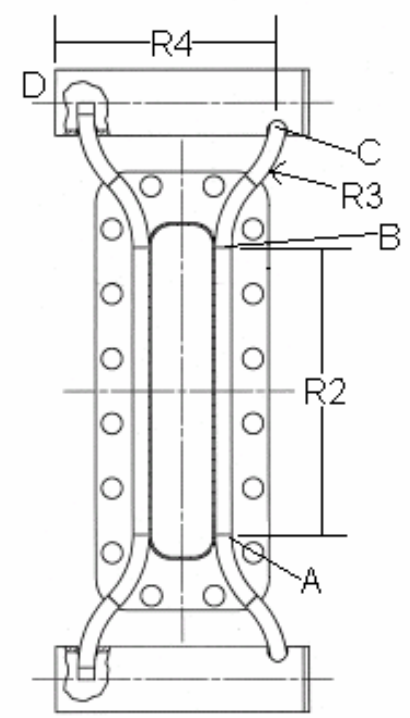

FIGURE 2. Section view of the waveguide used for thermal analysis.

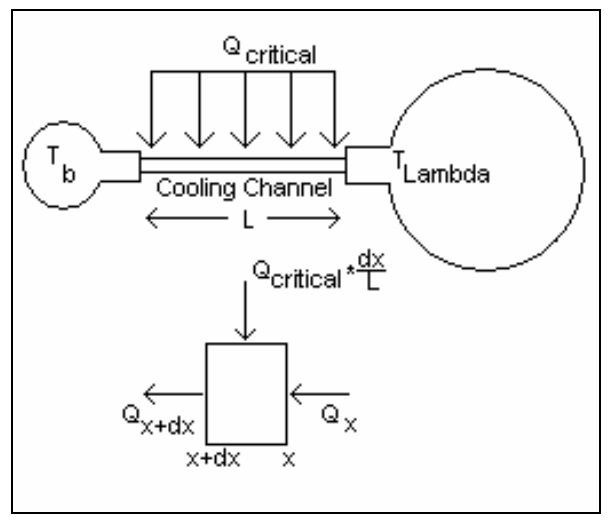

FIGURE 3. Energy Balance Diagram

equation was formed that more accurately describes the critical heat flux for R2 [3]. Figure 3 shows a diagram of the energy balance for a differential element of the channel.

The energy balance diagram in figure 3 can be expressed in equation form as:

$$
Q_{x}+\left(Q_{\text {critical }} \cdot \frac{d x}{L}\right)=Q_{x+d x}
$$

The heat rate equation used, equation (4), is for turbulent flow in wide channels [2]. Although it may not physically describe the flow in our design, it is a good approximation.

$$
Q_{x}=q_{x} \cdot A_{c}=A_{c}\left(\frac{d T}{d x} \frac{1}{f(T)}\right)^{\frac{1}{3}}
$$

Using Taylor series expansion and applying the heat rate equation, the applicable differential equation can be derived for the critical heat load [3]. 


$$
\frac{Q_{\text {critical }}}{A_{c}} \cdot \frac{d x}{L}=d\left(\frac{1}{f(T)} \cdot \frac{d T}{d x}\right)^{\frac{1}{3}}
$$

In equation (5):

$\mathrm{Q}_{\text {critical }}=$ uniform radial heat load along the length of cooling tube (watts)

$\mathrm{A}_{\mathrm{c}}=$ cross sectional area of cooling tube $\left(.37 \mathrm{~cm}^{2}\right)$

Integrating equation (5) yields an expression for the critical heat load for a channel exposed to a uniform heat load along its length [4].

$$
Q_{\text {critical }}=A_{c}\left(\frac{4}{L} \int_{T_{b}}^{T_{\lambda}} \frac{d T}{f(T)}\right)^{\frac{1}{3}}
$$

Equations (2) and (6) accurately describe the critical heat load for the different cooling tube sections. By applying the conservation of energy and making initial guesses for the temperatures at B and C, figure 2, an iterative method was used to satisfy equation (7) and solve for the critical heat load of the heat station.

$$
Q_{R 2}=Q_{R 3}=Q_{R 4}
$$

The analysis shows that the design will conduct away a total of .64, 1.12 and $1.52 \mathrm{~W}$ at cryogenic system operating temperatures $\left(\mathrm{T}_{\mathrm{b}}\right)$ of 2.1, 2.05 and $2.0 \mathrm{~K}$, respectively.

\section{TESTING}

To verify the thermal analysis and determine the operating characteristics of the $2 \mathrm{~K}$ heat station, two waveguides were instrumented with heaters, temperature diodes and pressure transducers. Figure 4 shows the instrumentation layout.

Tests were performed after commissioning in the FEL with the cryomodule set up for normal operating conditions. The test procedure was 1) record the steady state values of temperature and pressure, 2) introduce heater power to the heat station, 3) record the time for local pressure instabilities to occur, and 4) turn off the heater and allow the system to return to steady state conditions. The test procedure was repeated at different heater powers. During testing the pressure and temperature data were logged using JLab’s EPICs system.

Because no time constant data were available, testing was started at $5 \mathrm{~W}$ of heater power and lowered in $.5 \mathrm{~W}$ increments. The $5 \mathrm{~W}$ test run is shown graphically in figure 5 . Figure 5 shows the FPC temperature diodes respond quickly to the applied heater power. There was no measurable change in the waveguide temperatures. The FPC diodes were used as an indicator that the system had returned to steady state. In all cases the time needed for return to steady state was $\sim 45$ minutes. The pressure instability observed in figure 5 was measured at the pressure tap directly above the cooling tube. The instability was not observable at any other locations. Also, the cavity frequency was monitored for microphonic effects. None were observed. 


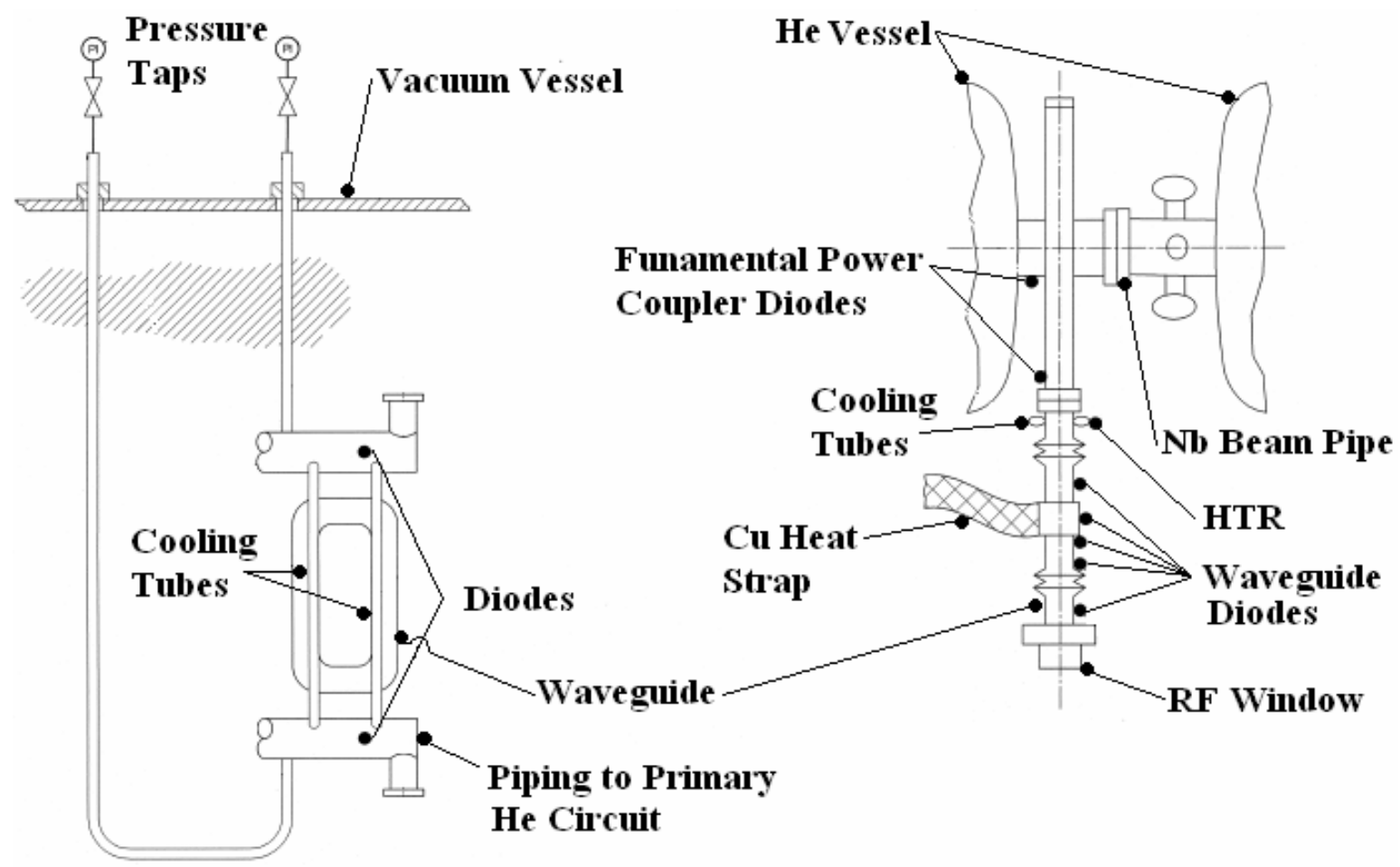

Elevation View

$\underline{\text { Plan View }}$

FIGURE 4. Instrumentation Layout

Waveguide \#4 Data (5W Heater Power)

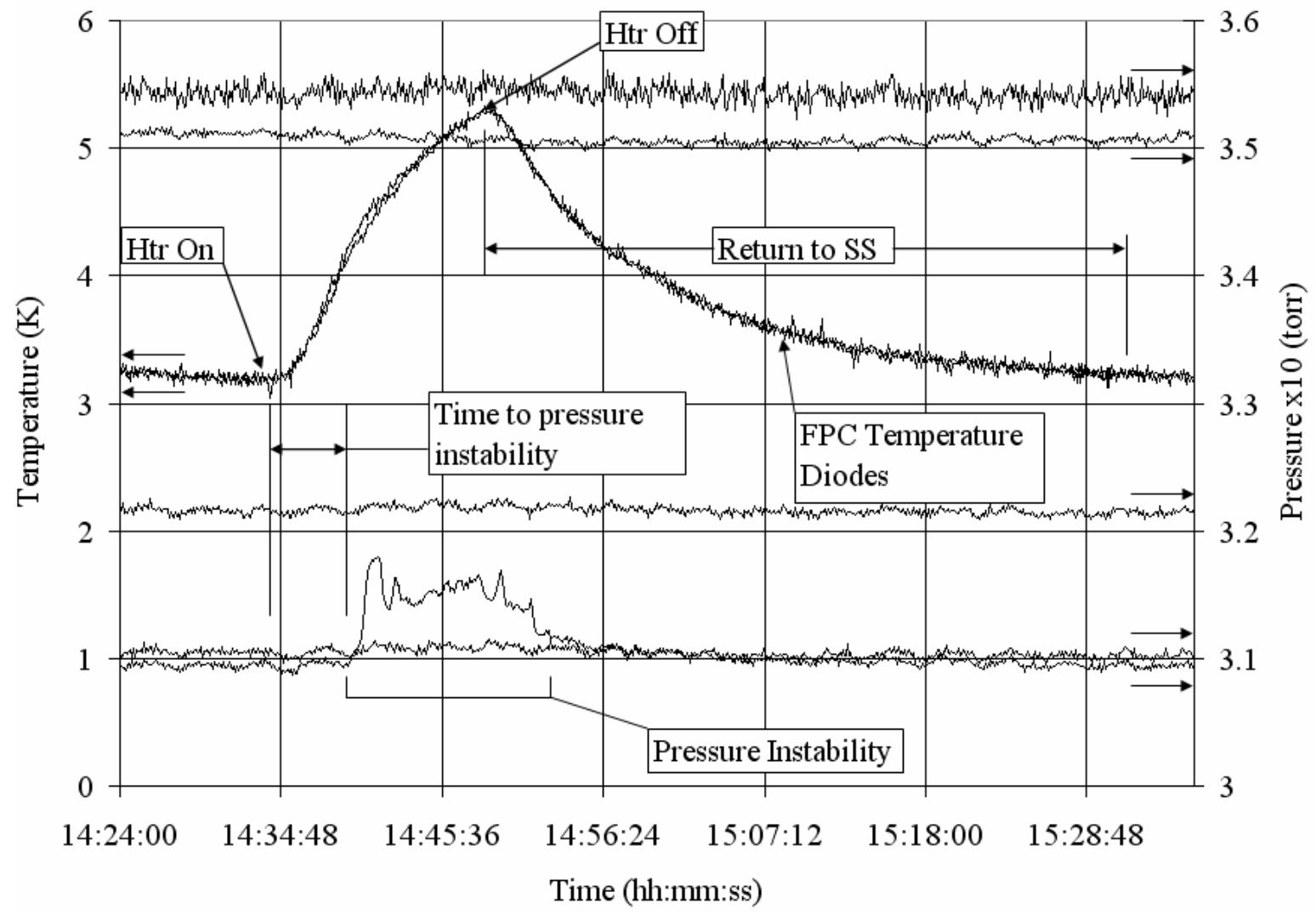

FIGURE 5. Logged temperature and pressure data for the test run at 5W of heater power. 


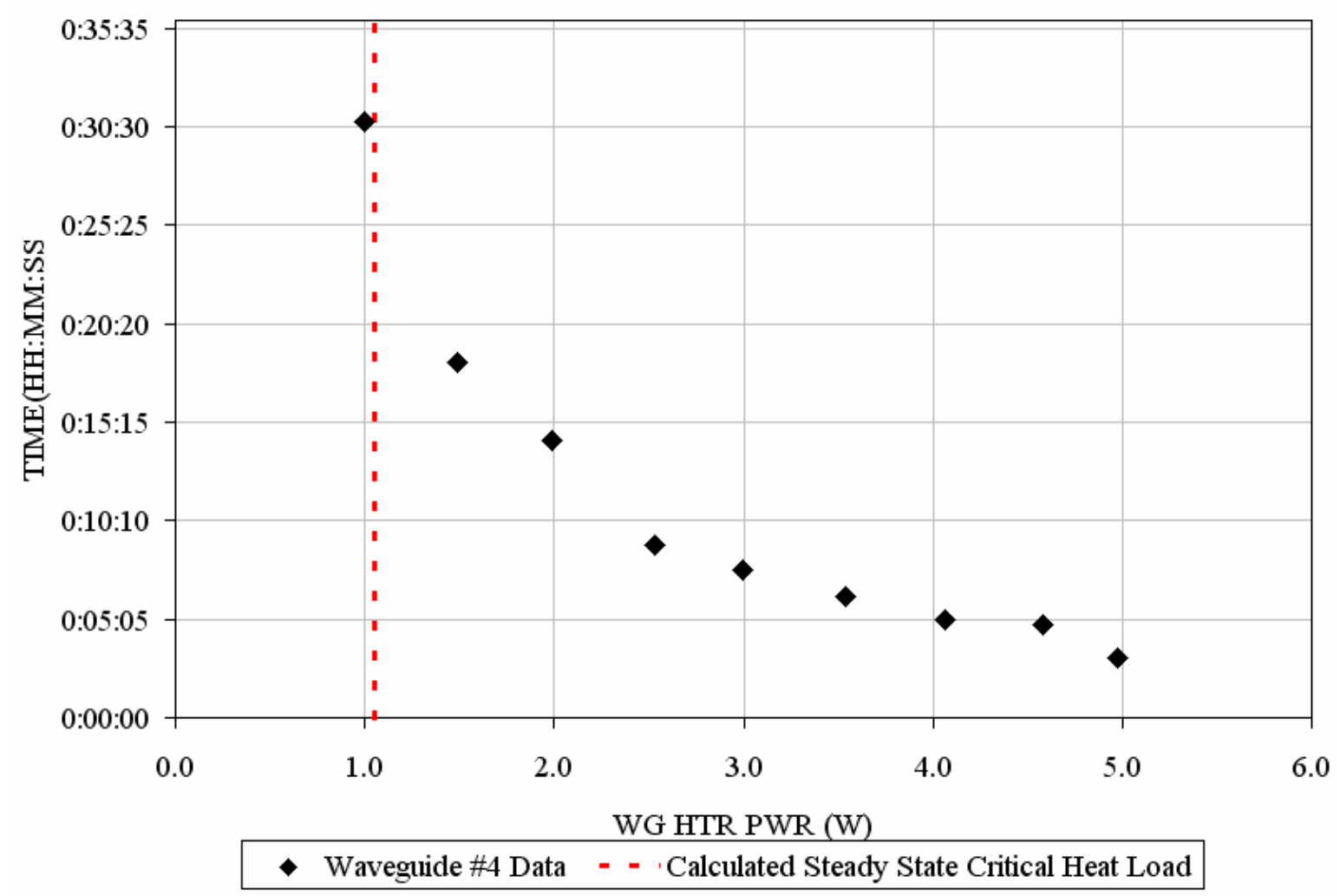

FIGURE 6. Test data for various heater powers at an operating pressure of 0.037atm.

Figure 6 shows the results of testing at various heater powers. Below $1 \mathrm{~W}$ no pressure instabilities were observed. The thermal model for a resistance heater input is different than the model for RF heat input. For the test configuration, heat is introduced at the center of the cooling tube. Equation 2 describes R2, R3 and R4 under test conditions. The return header pressure for this set of tests was $0.037 \mathrm{~atm}$. The corresponding bath temperature is $2.06 \mathrm{~K}$. The steady state critical heat load for the test configuration was calculated to be $1.05 \mathrm{~W}$ at a bath temperature of $2.06 \mathrm{~K}$. The dashed line in figure 6 represents the calculated steady state critical heat load. The difference in the calculated value and the test data can be attributed to the widely varying thermal properties of $\mathrm{He}$ II at cryogenic temperatures.

\section{SUMMARY}

A 2K superfluid helium heat station design was successfully incorporated in FEL03, an energy upgrade effort cryomodule. The design takes advantage of the extremely high thermal conductivity of He II. The results of testing are in good agreement with the thermal analysis. The local pressure instabilities caused by transition/turbulence in the cooling tubes had no measurable influence on cavity frequency. Also, the instabilities were not observable at any other locations within the cryomodule. It was later discovered that the unstable FPC temperatures were the result of the cavity turn on method. This was solved by changing the turn on procedure. Therefore, the heat station was not installed on Renascence nor planned for future cryomodules. Further testing of the 
$2 \mathrm{~K}$ heat station at various pressure levels within the primary helium circuit is dependent on facility and experimental schedules.

\section{REFERENCES}

1. Flynn, T. M., Cryogenic Engineering, Marcel Dekker, Inc., New York, 1997

2. $\quad$ Van Sciver, S. W., Helium Cryogenics, Plenum Press, New York, 1986

3. Incropera, F. P., DeWitt, D. P., Fundamentals of Heat and Mass Transfer, John Wiley \& Sons Inc., New York,1985

4. Daly, E. F., Kupiszewski, T., Christianson, O. R., "Thermal Analyses of the SMES ETM Power Bus Cryostat" in Advances in Cryogenic Engineering 39A, edited by Peter Kittel, Plenum Press, New York, 1994 